\title{
MYCOTIC KERATITIS CAUSED BY ASPERGILLUS FUMIGATUS*
}

\author{
BY \\ E. BALAKRISHNAN \\ Government Ophthalmic Hospital, Madras, India
}

IT is not uncommon to find infections of the cornea with varied organisms of the soil in South India, where agriculture is the main occupation of the people, and mycotic keratitis is often encountered in ophthalmic practice.

\section{Case Report}

A farmer aged 50 years was admitted to the Government Ophthalmic Hospital, Madras, on September 26, 1960, with pain and loss of vision of 3 days' duration in the right eye.

\section{Examination}

Right Eye: Corneal hypopyon ulcer. Visual acuity perception of light. Lacrimal passage free.

Left Eye: Visual acuity 6/6. Anterior segment and fundus normal.

The following routine treatment was given for one week: subconjunctival injections of crystalline penicillin 100,000 units, local atropine, terramycin ointment and bandage, and sulphadiazine $4 \mathrm{~g}$. daily in divided doses with alkalis. There was no improvement.

The case was then reviewed by the author and the following clinical picture was observed:

Right Eye: Corneal ulcer $6.5 \mathrm{~mm}$. diameter, faintly yellow with an irregular round border (Fig. 1). The centre of the ulcer was necrosed but the surrounding cornea was clear.

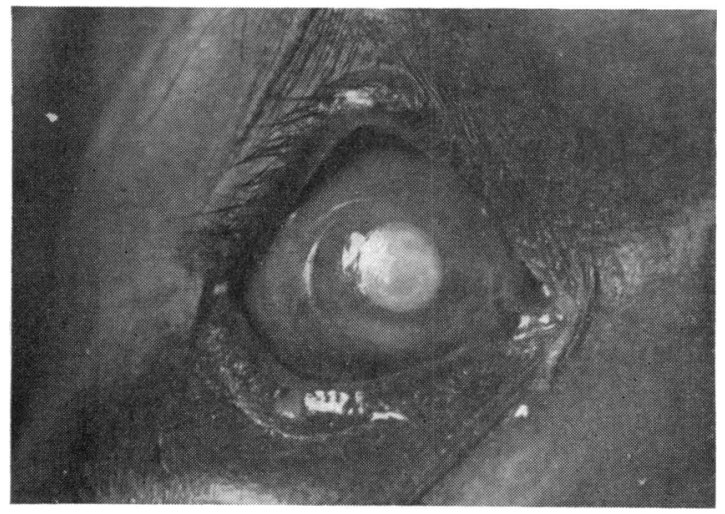

FIG. 1.-Appearance of corneal ulcer in right eye.

* Received for publication November 28, 1960. 
A small hypopyon occupied the bottom of the anterior chamber. Slip-lamp examination showed necrosis of the epithelial layers, with infiltration and swelling of Bowman's membrane and the substantia propria. Swollen tissues were arranged irregularly as round yellowish plaques one above the other. A few dark brown spots were seen on the surface in the centre, and the substantia propria was infiltrated around the ulcer. There was nothing characteristic in the hypopyon.

A fungal infection was suspected and a history of dust entering the right eye 2 days previously was elicited. The patient had applied gingelly oil from a open vessel, as a soothing agent, and next morning acute pain had developed with gross loss of vision.

The family history was negative, and chest $x$ rays, tests for venereal disease, and urine analysis showed nothing abnormal.

All local treatment was stopped and the eye was irrigated with normal saline for 3 days. On the 4th day, the ulcer was scraped and inoculated into Sabouraud's glucose medium, and Aspergillus fumigatus was grown on primary culture (Fig. 2).

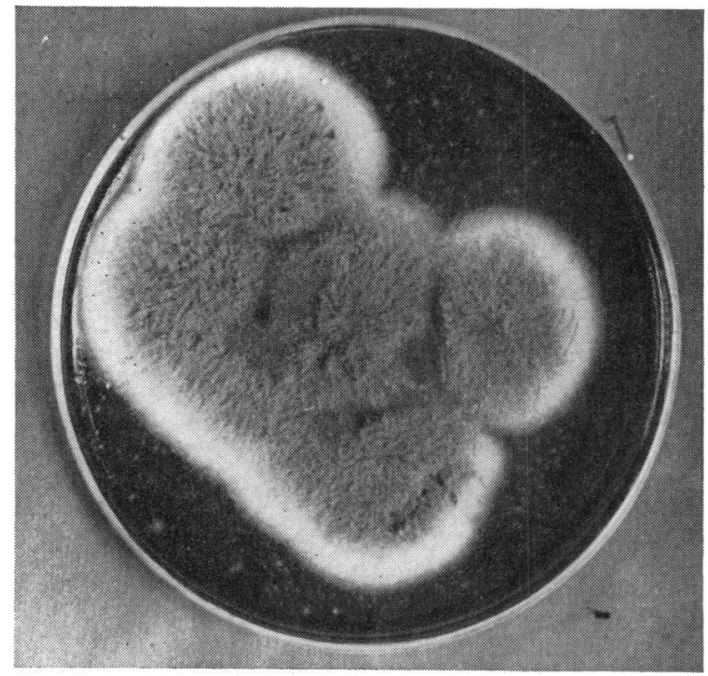

FIG. 2.-Culture of Aspergillus fumigatus.

Treatment.-Nystatin tablets (Mycostatin, Squibb, 1 tablet $=500,000$ units) were given 3 times a day for 8 days. No antifungal ophthalmic ointment was available. Atropine was applied locally once a day and the eye bandaged.

Result.-On the 2nd day after starting the treatment the hypopyon was absorbed, and on the 8th day the ulcer had healed completely leaving a dense leucoma, and the patient was completely relieved of pain. A slit-lamp examination on October 18,1960, showed a corneal leucoma with a faint yellow irregular margin. The centre of the leucoma appeared as a series of laminated white plaques with concentric rings, arranged one above the other. A small brownish area occupied the centre of the surface of the leucoma. The pupil was dilated because of the atropine, and the lens was cataractous and milky-white but could not be examined in detail because of the large leucoma. The ocular tension was normal, and the visual acuity had improved to hand movements. The patient was discharged with instructions to report after 4 weeks for surgical treatment. 


\section{Discussion}

The following points are of interest in this case:

(1) The source of infection with Aspergillus fumigatus may have been the dust or the oil from an open vessel.

(2) The ulcer healed completely with systemic Nystatin therapy and no antifungal ointment was used. Probably the Aspergillus fumigatus has an endotoxin, which was neutralized by the Nystatin.

(3) A complicated cataract developed within 20 days, suggesting that the fungus acted on the intra-ocular structures by its endotoxin.

It is important to include the possibility of fungal infection so as to institute proper treatment before the cornea is irreversibly damaged.

I should like to thank Mr. T. K. Ramachandra Reddy, for culturing the Aspergillus fumigatus, and Dr. T. T. Ramalingam, Superintendent of Government Ophthalmic Hospital, Madras, for permission to publish this case.

\section{BIBLIOGRAPHY}

Ainsworth, G. C. (1952). “Medical Mycology: An Introduction to its Problems”, p. 9. Pitman, London.

Anderson, B., Roberts, S. S., Jr., Gonzalez, C., and Chick, E. W. (1959). A.M.A. Arch. Ophthal., 62, 169.

BerLINER, M. L. (1949). "Biomicroscopy of the Eye", vol. 1, p. 531. Hoeber, New York.

ReEse, E. T., CReVETz, H., and MANDELS, G. R. (1955). Farlowia, 4, 409. 